\title{
El gobierno de Calderón Sol La evaluación de los salvadoreños
}

\author{
Instituto Universitario de Opinión Pública
}

\section{Resumen}

Coincidiendo con la finalización del período presidencial de Calderón Sol, el IUDOP de la UCA realizó una encuesta a mediados del mes de mayo para recoger y conocer la opinión de los salvadoreños sobre la segunda gestión arenera. La pesquisa, llevada a cabo a nivel nacional con una muestra de 1266 adultos, revela que el gobierno logró mejorar su imagen pública al final de su mandato aunque no pudo alcanzar los niveles de popularidad al inicio de su gestión, ello porque buena parte de los salvadoreños le reprochan el no haber terminado con la delincuencia y el haber favorecido más a los sectores poderosos del país.

\section{Introducción}

Hace cinco años, cuando Armando Calderón Sol asumió la presidencia de la república como sucesor de Alfredo Cristiani, ARENA y su presidente electo estaban en su mejor momento. Con un apoyo indiscutible de casi la mitad de los salvadoreños y un presidente elegido que venía con una imagen pública exitosa por su desempeño en la alcaldía de San Salvador. Un año después de haber tomado posesión, la opinión pública no era la misma: la población tenía una imagen muy negativa del nuevo presidente y en ese momento se comenzaba a gestar la tendencia que habría de apartar a los areneros de las alcaldías del AMSS y de varios curules en la Asamblea Legislativa. Al finalizar su mandato, la segunda presidencia de ARENA debe entregar nuevamente las riendas del país a uno más de sus correligionarios; sin embargo, en términos de imagen y opinión pública, esta transición no se compara con la que tomó lugar hace cinco años. Calderón Sol no se va ovacionado como se fue Cristiani en su momento y ARENA, aunque sigue siendo sin discusión la primera fuerza política del país, debe lidiar con una población que en definitiva prefiere trabajar o hacer cualquier cosa antes que pensar en política. Sin embargo, el segundo gobierno de ARENA tampoco se va vilipendiado, se va más bien dejando interrogantes en los ciudadanos antes que irritación y, sobre todo, 
se va dejando profundas dudas acerca de su legado.

El presente artículo es un intento por describir el estado de la opinión pública al finalizar el mandato de Calderón Sol. Para ello se concentra en presentar y analizar los resultados de la quinta encuesta de la serie de evaluación del gobiemo de Calderón Sol, serie que fue inaugurada en los primeros cien días de gobierno y contra la cual es posible contrastar algunos de los resultados que se presentan a continuación.

Lo que sigue a continuación no pretende ser el veredicto final sobre la gestión calderonista, constituye más bien la documentación de las imágenes, sentimientos y valoraciones de los salvadoreños sobre su primer gobierno de la posguerra. Para ello, el artículo desarrolla un apartado metodológico, el cual describe los pormenores de la investigación, y continúa con una serie de apartados que llevan un orden temático de las opiniones sobre el gobierno y el país durante el mismo: los problemas nacionales, los cambios positivos y negativos logrados por el gobierno de Calderón, la relación gobiemo-ciudadanía, la situación del país y la evaluación global del desempeño del gobiemo. Es necesario advertir que, por razones de espacio, ha sido necesario dejar fuera varios de los aspectos incluidos en el sondeo; sin embargo, lo más importante e ilustrastivo de la opinión pública recogida en el sondeo se ha intentado mostrar acá.

\section{Aspectos metodológicos}

Entre el 15 y el 18 de mayo de 1999, el Instituto Universitario de Opinión Pública (IUDOP), de la Universidad Centroamericana "José Simeón Cañas" (UCA), realizó una encuesta de opinión pública con el objeto de conocer lo que piensan los salvadoreños sobre el quinto año de gestión del gobierno de ARENA, el segundo año de las Alcaldías y de la Asamblea Legislativa, y a la vez recoger las expectativas ciudadanas sobre el nuevo gobierno.

El cuestionario empleado para llevar a cabo esta investigación se compuso básicamente de seis partes. En la primera se recogieron los datos demográficos de la persona. La segunda parte estuvo constituida por una batería de preguntas cuyo fin era conocer cómo evaluaban los salvadoreños el quinto año de gobierno de Calderón Sol. Para ello se solicitó a la persona entrevistada que dijera cuánto consideraba que el gobierno se había preocupado por combatir el problema que había señalado como el principal, y que evaluara la actuación del gobiemo de Calderón Sol a lo largo de toda su gestión. En esta parte también se dedicó un espacio para que los encuestados evaluaran, usando una escala de cero a diez, el trabajo de algunos Ministerios y expresaran cuánta capacidad para conducir el país creían que había tenido el gobierno y qué tan corrupto lo consideraban. Las interrogantes del tercer bloque estuvieron orientadas a conocer cómo evaluaron los salvadoreños la Asamblea Legislativa. La cuarta parte incluyó interrogantes respecto a la Alcaldía que le correspondía al entrevistado para evaluar la gestión de la misma en los últimos dos años. La quinta sección recolectó las expectativas de los ciudadanos respecto al nuevo gobiemo de Francisco Flores. Se preguntó sobre el principal reto que debería enfrentar, si debía continuar con la misma política económica y de seguridad pública, qué es lo primero que debía hacer para mejorar la situación del país, así como si creían que la situación del país mejoraría, empeoraría o seguiría igual con el gane de ARENA por tercera vez. Finalmente, las interrogantes del quinto bloque exploraron la confianza del entrevistado en ciertas instituciones, como la Asamblea Legislativa, la alcaldía de su localidad, la Policía Nacional Civil, la Iglesia Católica, etc. Y para terminar se preguntó sobre la opción de voto de las personas encuestadas. En este trabajo se presentan y analizan los resultados referidos a la evaluación del gobierno central, es decir, a lo que se relaciona con los cinco años de administración de Calderón Sol; el resto será abordado y presentado en publicaciones posteriores.

La muestra nacional se diseñó para representar lo más fielmente posible la totalidad de la población adulta salvadoreña, de acuerdo con los datos del Censo Nacional de Población de 1992 y del informe disponible de la Encuesta de Hogares de Propósitos Múltiples de la Dirección de Información del Ministerio de Economía de 1996. La cuota muestral incluyó 38 municipios de los catorce departamentos de la República. Como es usual en las encuestas del IUDOP, la muestra se dividió -en cada departamento- en dos grandes sectores: urbano y rural. El sector rural se dejó como una categoría aparte por las dificultades para encontrar criterios de tipificación en el campo. En el muestreo se consideraron también cuotas por sexo y edad de los encuestados, de tal manera que cada 
boleta se marcó con las características que debía cumplir el encuestado para ser considerado en la investigación.

La aplicación del cuestionario se hizo por aproximación no sistemática a los hogares ubicados en los municipios y las zonas definidas aleatoriamente. Los entrevistadores explicaron a las personas abordadas los objetivos y el tema de la encuesta. En cada caso se entrevistó únicamente a personas que quisieran contestar (una persona por hogar) y que cumplieran con los requerimientos descritos en la boleta para completar la cuota muestral.
La muestra final obtenida fue de 1266 encuestas válidas, con un margen de error estimado de $+/-0.04$ (cuatro por ciento). El 48.6 por ciento de los encuestados eran del sexo masculino y el 51.4 por ciento del sexo femenino. La edad promedio fue de 38.3 años con una desviación típica de 16.1 años. Los entrevistados dijeron tener un promedio general de 7.8 años de estudio. Por otro lado, el 49.8 por ciento estaban empleados en la actualidad o poseían un trabajo propio. En el Cuadro 1 se presenta el detalle de la muestra que se usó en el sondeo, según departamento de residencia y estrato socioeconómico del entrevistado.

Cuadro 1

Distribución de la población encuestada según departamento y sector social

\begin{tabular}{|c|c|c|c|c|c|c|c|c|c|}
\hline \multirow[b]{2}{*}{ Departamento } & & & \multicolumn{5}{|c|}{ Sector social } & \multicolumn{2}{|c|}{ Todos } \\
\hline & \multicolumn{2}{|c|}{ Alto } & Medio-alto & Medio-bajo & Obrero & Marginal & Rural & $\mathbf{N}$ & $\%$ \\
\hline \multirow[t]{2}{*}{ Todos } & $\%$ & 7.5 & 13.8 & 13.6 & 19.5 & 12.7 & 32.9 & $\longrightarrow$ & 100.0 \\
\hline & $N$ & 95 & 175 & 172 & 247 & 161 & 416 & 1266 & $\longrightarrow$ \\
\hline Ahuachapán & & 3.2 & 2.9 & 2.3 & 2.8 & 2.5 & 10.6 & 67 & 5.3 \\
\hline Santa Ana & & 7.4 & 7.4 & 7.6 & 8.1 & 8.1 & 10.1 & 108 & 8.5 \\
\hline Sonsonate & & 4.2 & 6.9 & 7.0 & 6.5 & 6.2 & 8.4 & 89 & 7.0 \\
\hline La Libertad & & 10.5 & 8.6 & 9.9 & 9.3 & 9.3 & 12.0 & 130 & 10.3 \\
\hline Chalatenango & & 4.2 & 2.3 & 3.5 & 2.8 & 3.1 & 5.5 & 49 & 3.9 \\
\hline San Salvador & & 33.7 & 37.1 & 38.4 & 37.2 & 38.5 & 11.8 & 366 & 28.9 \\
\hline Cabañas & & 3.2 & 4.0 & 1.7 & 2.0 & 1.9 & 3.8 & 37 & 2.9 \\
\hline Cuscatlán & & 3.2 & 2.9 & 3.5 & 3.2 & 3.1 & 4.1 & 44 & 3.5 \\
\hline San Vicente & & 4.2 & 2.9 & 1.7 & 2.4 & 2.5 & 3.4 & 36 & 2.8 \\
\hline $\mathrm{La} \mathrm{Paz}$ & & 5.3 & 5.7 & 5.2 & 4.5 & 5.0 & 4.8 & 63 & 5.0 \\
\hline Usulután & & 8.4 & 4.6 & 4.7 & 4.9 & 5.0 & 8.2 & 78 & 6.2 \\
\hline San Miguel & & 6.3 & 8.6 & 8.7 & 8.9 & 8.7 & 5.3 & 94 & 7.4 \\
\hline Morazán & & 1.1 & 2.3 & 2.3 & 2.4 & 2.5 & 5.8 & 43 & 3.4 \\
\hline La Unión & & 5.3 & 4.0 & 3.5 & 4.9 & 3.7 & 6.3 & 62 & 4.9 \\
\hline
\end{tabular}

\section{El problema principal del país}

Como es habitual en todos los sondeos del IUDOP, se pidió a los ciudadanos que identificaran el problema nacional más importante. A pesar de que ésta es una de las preguntas más frecuentes en las encuestas de opinión pública y las mismas muestran una constante en las percepciones ciudadanas sobre ese tema, el valor de incluir este aspecto tiene que ver con el hecho de evaluar si los cinco años de la administración Calderón Sol lograron modificar la percepción acerca de los pro- blemas fundamentales del país; en otras palabras, se trata de considerar si la gestión gubernamental logró reducir la preocupación por los problemas existentes hace cinco años y si provocó la aparición de otros problemas.

Los resultados de la encuesta, hecha en mayo de 1999, no revelan una percepción sobre los problemas especialmente diferente de lo que ha sido la constante en la última década (ver Coleman, Cruz y Moore, 1996). El gobiemo de Armando Calderón Sol cierra su período con una sociedad 
preocupada por la delincuencia, el desempleo, la pobreza, la economía en general y la inflación. Éstos son prácticamente los mismos problemas que definían y enfrentaban los salvadoreños hace cinco años (ver Cuadro 2), pero con un orden distinto de acuerdos y prioridades. En mayo de 1994, días antes de que Calderón Sol asumiera la presidencia de la república, el principal problema singular era ya la delincuencia; sin embargo, los problemas de carácter económico como la pobreza, el desempleo, la inflación sumaban más del 50 por ciento de las declaraciones ciudadanas y constituía la fuente de preocupación más común de los salvadoreños. Cinco años después, el segundo gobierno de ARENA deja una sociedad que aún percibe los mismos problemas, pero en proporciones diferentes; en concreto, deja una población aún más preocupada por la delincuencia - el porcentaje de personas preocupadas por la criminalidad casi se duplicó-, pero comparativamente mucho menos inquieta por los aspectos económicos que, no obstante, no han dejado de aparecer. Y los aspectos económicos mismos no tienen ahora el mismo peso: aunque todos los problemas de índole económico son ahora menos señalados que antes, la percepción de la inflación o el alto costo de la vida como problema prácticamente desapareció y la preocupación por la economía en general se redujo a casi la mitad.

\section{Cuadro 2}

Principales problemas del país al inicio y al final de la administración Calderón Sol (En porcentajes)

\begin{tabular}{lcc}
\hline Problema & \multicolumn{2}{c}{ Fecha del sondeo } \\
& $\begin{array}{c}\text { Mayo de } \\
\text { Mayo de } \\
1994\end{array}$ & 1999 \\
\hline Delincuencia & 35.6 & 61.1 \\
Desempleo & 14.0 & 10.9 \\
Economía en general & 14.0 & 6.7 \\
Pobreza & 13.5 & 10.3 \\
Alto costo de la vida & 12.2 & 1.7 \\
Otros problemas & 8.0 & 6.9 \\
No sabe & 0.0 & 2.4 \\
\hline
\end{tabular}

Fuente: IUDOP, Boletín de prensa No. 3, Año IX; scrie de informes No. 76.
Estos datos sugieren, según las declaraciones de la población, que el gobierno saliente deja un país con un problema mucho mayor de delincuencia que el que recibió en 1994, pero con unas dificultades económicas $\longrightarrow$ al menos una preocupación por las mismas - mucho menores, sobre todo en el ámbito de la inflación y el alto costo de la vida. Con todo, ninguno de los problemas fundamentales desapareció, pero tampoco se destacó uno nuevo. El gobierno en cuestión va dejando tras de sí una configuración distinta de los mismos problemas que recibió hace cinco años.

Sin embargo y al preguntar directamente, la mayor parte de la población consultada piensa que el gobiemo de Calderón Sol se preocupó nada o poco por resolver esos problemas fundamentales del país señalados con anterioridad, esto es, alrededor del 67 por ciento. Los datos muestran que estas opiniones son aún mayores entre aquéllos que señalaron los problemas de índole económi-
El gobierno de Armando Calderón Sol cierra su período con una sociedad preocupada por la delincuencia, el desempleo, la pobreza, la economía en general y la inflación. Éstos son prácticamente los mismos problemas que definían y enfrentaban los salvadoreños hace cinco años... ca ( 74.3 por ciento) que entre quienes señalaron a la delincuencia (65.3 por ciento); es decir, que las personas más preocupadas por el desempleo, la pobreza, la inflación y la economía en general piensan con más frecuencia que el gobierno no se esforzó por combatir esos problemas. De alguna manera, quienes señalan a la economía como problema nacional tienden a ser más críticos con respecto al desempeño del gobierno que aquéllos que no refieren este problema.

Establecido este marco de opiniones sobre los problemas nacionales, hay que ver, entonces, cómo los salvadoreños evalúan directamente al gobierno en toda su gestión.

\section{Lo positivo del gobierno}

Para comenzar, el sondeo de la UCA revela que seis de cada diez salvadoreños (el 61.1 por ciento) percibieron cambios positivos en el país desde que comenzó a gobernar el presidente Calderón Sol. Esta opinión es mayor en las zonas metropolitana y central del país, pero es menor en las zonas más periféricas (occidente y oriente); además es una opinión que aumenta en los estratos 
socioeconómicos más altos (el 56 por ciento de los obreros ve cambios positivos frente a un 74.7 por ciento que se observa en los sectores altos). En otras palabras, las mejores opiniones en el país son más percibidas por aquellas personas que residen en la metrópoli y que forman parte del sector más favorecido de la población.

Esta opinión positiva sobre los cambios del país constituye el nivel más alto alcanzado por el gobierno en toda su gestión. En las anteriores evaluaciones anuales del desempeño del gobierno, el porcentaje de personas que afirmaban haber percibido cambios positivos en el país nunca superó el 45 por ciento (ver IUDOP, 1995a; IUDOP. 1996; IUDOP, 1997; IUDOP, 1998) y

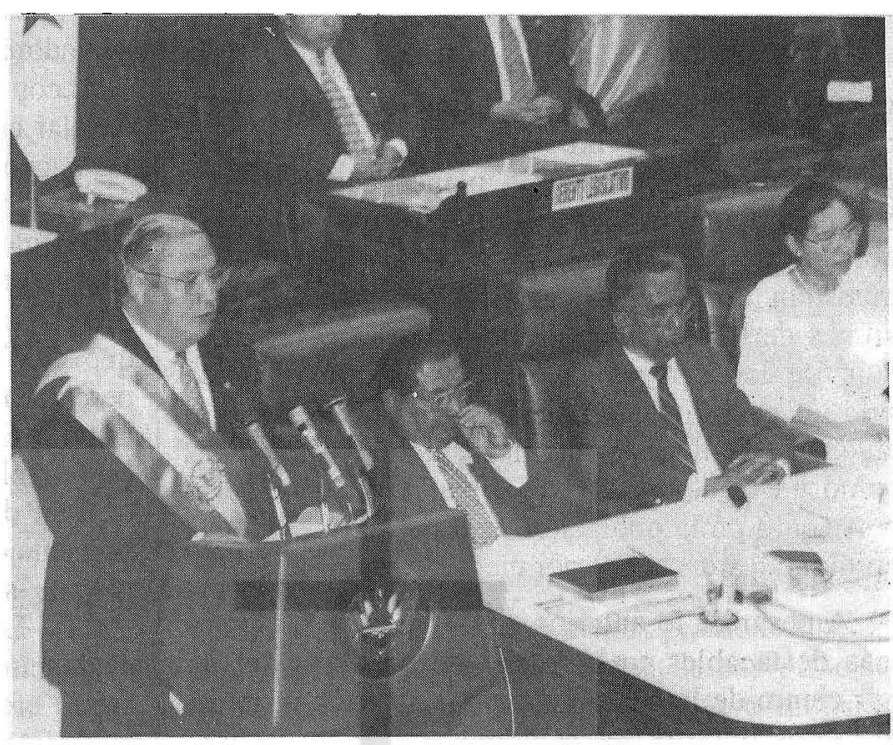
en la mayor parte de ellas sólo una tercera parte de los salvadoreños veían cambios positivos con el gobiemo; no es sino hacia el final de su gestión cuando los ciudadanos logran percibir mejoras en el país. Esto probablemente se debe al hecho de que muchas de las iniciativas gubernamentales no comenzaron a dar frutos perceptibles sino hasta después de algún tiempo y.al esfuerzo innegable de la administración Calderón Sol por mejorar su imagen ante el público, en los últimos tres meses de gestión, por medio de la inauguración de obras de infraestructura.

Figura 1

Opinión sobre el principal logro del gobierno de Calderón Sol a lo largo de su gestión

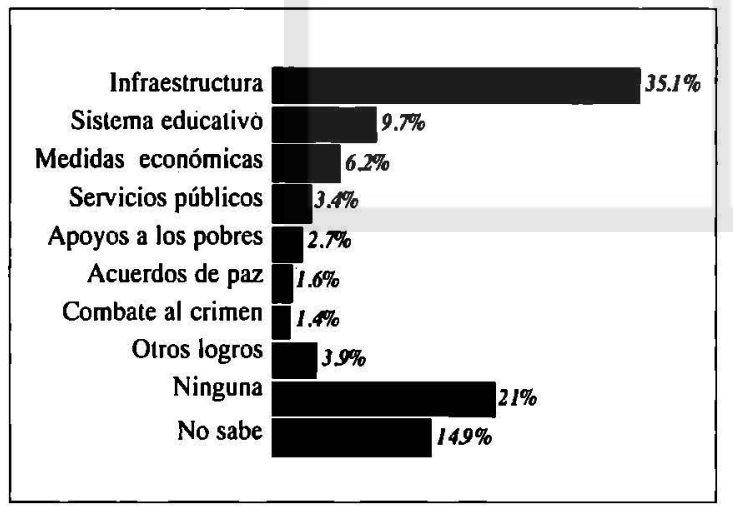

De hecho, el principal logro que buena parte de la ciudadanía atribuyó al gobierno de Armando Calderón Sol tiene que ver con eso: el 35.1 por ciento de los ciudadanos afirmaron que el logro o éxito fundamental de la segunda administración de ARENA fue la construcción de infraestructura vial y general del país, muy por encima del casi 10 por ciento que señaló las mejoras en la educación o el 6 por ciento que se refirió a la política económica, entre otras cosas. A pesar de ello, un porcentaje importante de la población declaró que no existía logro alguno en la gestión Calderón y un 14 por ciento no supo identificarlo, lo que conlleva a pensar que una tercera parte de la población no supo definir los logros del gobierno saliente.

El esfuerzo del gobierno saliente por promover su imagen a través de la construcción de obras de infraestructura parece haber dado resultado. Ahora bien, la consideración de la infraestructura como un cambio positivo parece estar relacionada con la zona de residencia del entrevistado. Las personas que viven en el Área Metropolitana de San Salvador (AMSS) y aquéllas que habitan en las zonas urbanas fueron las que más señalaron a la construcción de obras físicas como el principal logro del gobierno, frente a los habitantes de zonas como el oriente del país y las áreas rurales, quienes tendieron a valorar menos la infraestructura. Esta tendencia en los datos es lógica si se toma en cuenta que el programa de construcción de obras civiles por parte del gobierno se concentró esen- 
cialmente en las áreas urbanas metropolitanas.

Otros aspectos recibieron mucho menos atención por parte del público. Las mejoras en el sistema de educación - probablemente uno de los esfuerzos más sistemáticos y planeados de toda la administración Calderón Sol- fueron valoradas por uno de cada diez salvadoreños y los datos no muestran diferencias significativas entre las opiniones de los distintos grupos sociales con excepción de los campesinos, que valoraron un poco más los cambios en el sistema educativo. Por otro lado, las medidas económicas fueron reconocidas como un cambio positivo por un poco más del 6 por ciento de la población total del país, pero esta opinión alcanza el 9.3 por ciento en el AMSS.

A pesar de lo anterior, quizás una de las cosas más destacables es el hecho de que más del 35 por ciento de la gente no supo o no pudo atribuirle logros importantes al gobiemo por su gestión. Esta respuesta fue significativamente más común entre las personas que viven en las zonas periféricas del país (por ejemplo, la zona oriental), que habitan en el área rural y que tienen muy poca o ninguna formación educativa. Esta distribución de los resultados sugiere que las personas menos aventajadas en el ordenamiento socioeconómico del país fueron las que no lograron percibir éxitos en la gestión Calderón, probablemente porque los esfuerzos del mismo no se enfocaron en tales personas o porque, al menos, la gente lo percibió así1.

\section{Lo negativo del gobierno}

Del otro lado de la moneda se preguntó al público salvadoreño por los cambios negativos que ha experimentado el país desde que Calderón Sol asumió la presidencia de la república. En este caso, las opiniones se encuentran más divididas: el 52 por ciento de los consultados dijo haber percibido algún cambio negativo en el país en los últimos cinco años, mientras que el 44.5 por ciento dijo que no ha visto cambios negativos en el país. Esta opinión no se contradice con la anterior referida a los cambios positivos; una persona puede percibir aspectos positivos del desempeño gubernamental, pero ello no le impide, si se es objetivo, advertir los aspectos negativos de la actuación gubemamental.
A pesar de que un poco más de la mitad de los salvadoreños han notado cambios negativos en el país con la gestión del nuevo gobierno, hay que señalar que una comparación con los resultados de las encuestas de evaluación anteriores muestra que anteriormente el gobierno solía recibir más opiniones negativas. Por ejemplo, al concluir su primer año de gestión, la encuesta de evaluación gubemamental correspondiente (IUDOP, 1995a) señaló que el $\mathbf{7 7 . 3}$ por ciento de los ciudadanos habían percibido cambios negativos en el país desde que asumió el poder el segundo gabinete de ARENA; este nivel de desaprobación se mantuvo alto hasta el final de los cuatro años de gobiemo cuando el porcentaje de gente que veía cambios negativos en el país se redujo a un 60 por ciento (IUDOP, 1998). Los resultados de la última encuesta de evaluación señalan que esta opinión negativa sobre la efectividad del gobierno disminuyó sustancialmente al final de su mandato, lo cual contribuyó al mejoramiento de su imagen pública.

\section{Cuadro 3}

¿Ha notado algún cambio negativo en el país desde que entró Calderón Sol como presidente? (En porcentajes)

\begin{tabular}{lccc}
\hline & \multicolumn{2}{c}{ Respuesta } & \\
\cline { 2 - 3 } Variables & No & Sí & $\begin{array}{c}\text { No } \\
\text { sabe }\end{array}$ \\
\hline Todos & 44.5 & 52.0 & 3.5 \\
\hline Estrato & & & \\
Alto & 38.9 & 57.9 & 3.2 \\
Medio-alto & 36.0 & 61.7 & 2.3 \\
Medio-bajo & 34.3 & 64.5 & 1.2 \\
Obrero & 44.9 & 53.0 & 2.0 \\
Marginal & 47.2 & 48.4 & 4.3 \\
Rural & 52.4 & 42.1 & 5.5 \\
& & & \\
Estudios & & 43.4 & 5.0 \\
Ninguno & 51.6 & 48.2 & 5.1 \\
Primaria & 46.7 & 47.1 & 3.7 \\
Plan básico & 49.2 & 47.1 & 2.4 \\
Bachillerato & 47.8 & 49.8 & 1.1 \\
Superior & 31.5 & 67.4 & \\
\hline CHanotado algunn & & & \\
\hline
\end{tabular}

¿Ha notado algún cambio negativo en nuestro país desde que entró Calderón Sol como presidente?

1. Al respecto, la encuesta abordó el tema de quién es el grupo más favorecido por la gestión gubernamental. Los datos, que se presentan más adelante en este artículo, señalan que el gobierno se preocupó más por los "ricos". 
A preguntárseles sobre los fracasos del gobierno en toda su gestión, los ciudadanos respondieron de manera muy dispersa; es decir, no concentraron mucho sus respuestas en un solo fracaso sino que se mencionaron varios con frecuencias muy parecidas. De acuerdo con un poco más de la quinta parte de los salvadoreños (el 22.2 por ciento), el principal malogro de la administración Calderón Sol fue el incremento de la delincuencia, lo cual es consistente con las opiniones que colocan a la criminalidad como el principal problema del país y con las que señalan que el gobierno no se preocupó por controlar esa problemática. Otros fracasos atribuidos al gobierno tienen que ver con aspectos económicos: la inflación o el alto costo de la vida (9.4 por ciento), la pobreza (7.8 por ciento) y el desempleo (6.2 por ciento). En total, alrededor de un 23 por ciento de los consultados advirtió aspectos económicos como fracasos. Atención especial merece el hecho de que cerca del 10 por ciento de los salvadoreños señalen a la inflación como un fracaso, a pesar de que este tema aparece muy poco como problema fundamental del país. Una explicación a esta aparente contradicción tiene que ver con que algunas personas probablemente están haciendo un recuento de toda la administración y están señalando que el costo de la vida subió durante estos cinco años, a pesar de que en el último año se haya reducido un poco la tendencia hacia el encarecimiento de la vida.

Otras fallas mencionadas fueron la incapacidad de gobernar del gobierno (5.5 por ciento), la privatización de las empresas públicas (3.4 por ciento) y el incumplimiento de las promesas realizadas en la campaña electoral y en el programa original de gobiemo (2.8 por ciento). Un dato que vale la pena resaltar es el de la privatización de las empresas públicas como fracaso de la gestión gubernamental, según la opinión de una parte de los salvadoreños. La privatización fue una de las políticas más decididas y planificadas por el gobierno que concluye, el cual invirtió mucho de sus es-

fuerzos para hacer pasar a manos privadas las telecomunicaciones y la distribución de energía eléctrica; sin embargo, este empeño de la administración Calderón fue realizado, en buena medida, en contra de la opinión pública salvadoreña. Su aparecimiento como fracaso, pues no es fortuito y aunque el porcentaje de personas que lo mencionaron como tal es reducido, expresa la existencia de una desaprobación mayor. De hecho, el sondeo de evaluación del gobierno indicó que el 60 por ciento de los ciudadanos está en desacuerdo con el proceso de privatización que impulsó el gobierno saliente ${ }^{2}$.

Otro detalle que traen a cuenta las opiniones sobre los fracasos del gobierno es el tema del cumplimiento de las promesas por parte de la administración. Aunque sólo un poco menos del 3 por ciento lo mencionó como una de las principales fallas de la administración saliente, cuando se les preguntó directamente a los ciudadanos sobre ello, el 31.8 por ciento dijo que la segunda administración de ARENA no cumplió con sus promesas, frente a un 7.2 por ciento que sostuvo que sí las cumplió y el 59.7 por ciento que afirmó que sólo algunas. En otras palabras, sólo una pequeña parte de la población se siente totalmente satisfecha por el cumplimiento de los ofrecimientos electorales, el resto tiene algún grado de insatisfacción. Los mismos datos del sondeo revelan que las opiniones sobre el cumplimiento de promesas por parte del gobierno se encuentran estrechamente relacionadas con la evaluación general que los ciu-

2. En realidad, el apoyo a la privatización durante la gestión de Calderón Sol fue siempre muy bajo y la desaprobación muy alta. Más aún, las encuestas del IUDOP revelan que el rechazo a la misma ha crecido paulatinamente en los últimos años (ver IUDOP, 1996; IUDOP, 1997). 
dadanos hacen sobre el desempeño del gobiemo. Aquellas personas que piensan que la presidencia no cumplió sus promesas valoran muy mal el desempeño del gobiemo mismo; en cambio, quienes creen que la administración logró cumplir con algunas de sus promesas evalúan bien al gobierno y mejor aún quienes se declaran satisfechos por el cumplimiento de las promesas gubermamentales.

\section{Figura 2}

Opinión sobre el cumplimiento de promesas por parte del gobierno de Calderón Sol

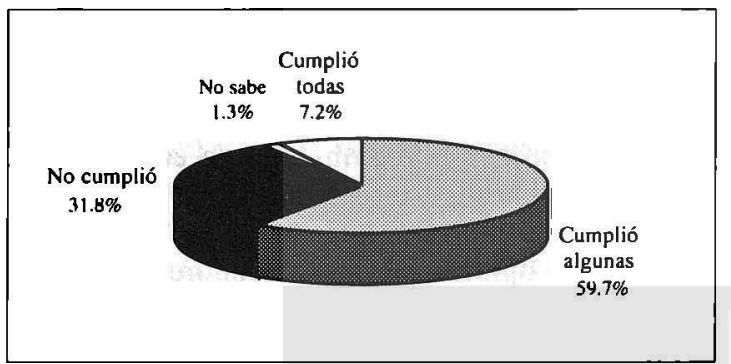

¿Cree usted que el actual gobierno de ARENA cumplió con sus promesas?

Estos datos muestran el peso de las expectativas sobre la evaluación general del desempeño del gobierno. Esto es, en la medida en que las personas ven satisfechas sus expectativas, por muy diversas y disímiles que éstas sean, en esa medida tendrán una valoración mejor del gobierno que aquéllos que sienten que las promesas no se cumplieron. En este punto juega un papel muy importante la percepción de las relaciones entre el gobierno y las personas; es decir, ¿cómo los salvadoreños valoran su vínculo con la administración en términos de beneficios y de atención?

\section{La relación gobierno-ciudadanos}

La encuesta de la UCA incluyó dos ítemes para evaluar la percepción de los ciudadanos sobre qué tanto se ha interesado y relacionado eficientemente el gobierno con ellos. En concreto se pidió a los consultados que expresaran su acuerdo o desacuerdo sobre las siguientes dos frases: (a) Las acciones del gobierno han beneficiado a personas como usted, y (b) Personas como usted influyeron en las decisiones del gobierno. Los resultados muestran que la mayor parte de la gente piensa que el gobierno no la benefició (69.3 por ciento) y, más aún, piensan que ellos como ciudadanos no pudieron influir en las decisiones del mismo (74.2 por ciento). Lo anterior significa que el gobierno no logró generar una percepción de efícacia política en su relación con la ciudadanía. Los resultados son todavía más elocuentes cuando se analizan en función del estrato social (ver Cuadro 4). De acuerdo con dicho cuadro, las personas que se encuentran en estratos socioeconómicos más altos son las que más sienten que el gobierno las ha beneficiado y que ellas han influido en él; mientras tanto aquéllos que se encuentran en los estratos socioeconómicos más "bajos" de la sociedad salvadoreña se sienten más separados del gobierno. Estos datos coinciden con la tendencia que muestra que los estratos más bajos han tenido más dificultades para encontrar cambios positivos en la sociedad en la administración Calderón Sol, ello probablemente porque las acciones del gobiemo no se han concentrado en beneficiar a esos sectores, los cuales, en su mayoría, tampoco se han sentido tomados en cuenta.

\section{Cuadro 4}

Personas que están de acuerdo con que el gobierno los benefició y con que ellos influyeron en el gobierno, según estrato socioeconómico (En porcentajes)

$$
\text { Ítem }
$$

\begin{tabular}{l|c|c} 
Estrato & $\begin{array}{c}\text { Las acciones del } \\
\text { gobierno han } \\
\text { beneficiado } \\
\text { a personas } \\
\text { como Ud. }\end{array}$ & $\begin{array}{c}\text { Personas como } \\
\text { Ud. influyeron } \\
\text { en las } \\
\text { decisiones } \\
\text { del gobierno }\end{array}$ \\
\hline Todos & 23.8 & 17.4 \\
\hline Alto & 36.9 & 28.7 \\
Medio-alto & 29.7 & 24.7 \\
Medio-bajo & 27.9 & 20.3 \\
Obrero & 20.3 & 10.6 \\
Marginal & 18.8 & 13.2 \\
Rural & 20.6 & 16.3 \\
\hline
\end{tabular}

Otro dato de la encuesta de evaluación gubernamental que confirma esa tendencia es el que muestra las opiniones sobre qué tanto el gobierno saliente escuchó las demandas de la población. Los resultados indican que el 42.4 por ciento de los consultados piensan que la administración Calderón Sol escuchó poco las demandas de la pobla- 
ción, a ello se suma un 20.2 por ciento que dijo que el gobiemo no ha escuchado "nada" de las demandas populares. Sólo un 10.1 por ciento sostuvo que el gobierno se preocupó "mucho" por atender las demandas de la población y un 23.7 por ciento que dijo que se preocupó "algo". Las personas que perciben que el gobierno escuchó más las demandas son aquéllas que ocupan la posición más alta de la escala socioeconómica; en cambio, entre los sectores obreros y marginales, las opiniones predominantes son que la segunda presidencia arenera escuchó poco o nada a la población.

Esta fue una constante en la opinión pública sobre el gobiemo de Calderón Sol. A lo largo de su gestión, buena parte de los salvadoreños señalaron repetidamente que el gobierno de turno no estaba poniendo atención a las necesidades de la ciudadanía; más aún, muchos de ellos sentían que su gestión estaba decididamente orientada a favorecer a grupos específicos de la sociedad por encima de asistir a las mayorías del país.

Cuando a los ciudadanos se les preguntó si el gobiemo de Armando Calderón Sol se preocupó por todos los salvadoreños o si existió un grupo que recibió más atención de la presidencia que otros, las respuestas se volcaron de manera elocuente. Sólo el 19.5 por ciento afirmó que el gobierno benefició a todos los salvadoreños por igual y un 2.2 por ciento mencionó a los pobres como el grupo más favorecido por la gestión arenera; el resto de ciudadanos consignó a los grupos de poder como los más privilegiados por el gobierno: el 40.8 por ciento sostuvo que los ricos, las personas con dinero, fueron los más beneficiados; el 18.2 por ciento indicó que el gobiemo mismo o los integrantes de la administración fueron los más favorecidos por su propia gestión; el 9.6 por ciento señaló a los empresarios ${ }^{3}$ y un 4.3 por ciento citó a los miembros del partido ARENA como el grupo más beneficiado por las políticas del gobierno arenero.

Como ya se ha señalado antes, esta impresión sobre la administración Calderón Sol no es nueva, no aparece en las últimas etapas de su mandato. La primera encuesta de evaluación anual del gobierno, realizada en mayo de 1995 , mostraba ya que alrededor del 54 por ciento de los salvadoreños pensaban que el gobierno estaba apoyando sólo a los ricos y a los empresarios; en tanto que un 16.4 por ciento sostenía que la presidencia respaldaba a todos los salvadoreños por igual. Cuatro años después, la opinión de la gente concedió un poco menos de interés al gobiemo por los más poderosos, pero no cambió la orientación fundamental de su opinión acerca de la vocación gubernamental por los "ricos".

Y es que detrás de estas opiniones está la percepción de que las políticas del gobierno, especialmente las económicas, no favorecieron a la mayoría de la población. En un sondeo realizado a inicios de 1995, cuando el gobierno ahora saliente anunció su paquete de medidas económicas, se encontró que seis de cada 10 salvadoreños pensaban que el plan económico impulsado por el gobierno beneficiaría solamente a los más ricos, y que más del 60 por ciento de los ciudadanos pensaba que tal plan no mejoraría la situación económica del país y de los ciudadanos (IUDOP, 1995b). Esta ha sido una opinión que a grandes líneas tampoco revirtió su tendencia fundamental después de cuatro años y que remite a otro cúmulo de opiniones sobre el desempeño del gobierno y la situación del país.

\section{La situación del país después de cinco años de Calderón Sol}

Éste es un tema que ya comenzó a ser abordado con los cambios positivos y negativos en el país desde que Calderón Sol asumió el poder, en junio de 1994. Un análisis más complejo ${ }^{4}$ de esos resultados expuestos en apartados anteriores permite dividir a la población en cuatro grupos de opinión. Por un lado están quienes no han percibido cambios de ningún tipo en el país, ni positivos ni negativos, con la gestión calderonista; éstos suman el 16.4 por ciento de la población. Por otro lado, se encuentran quienes sólo percibieron cam-

3. Esta categoría es, para mucha gente, muy cercana a la categoría semántica de los "ricos". Sin embargo, se dejó como una calegoría separada pues muestra el énfasis del contestante por identificar un grupo más específico que aquéllos.

4. En concreto se combinaron las respuestas de las preguntas sobre los cambios positivos y negativos del país. Se crearon cuatro calegorías distintas para diferenciar aquéllos que no percibieron cambios de los que percibieron sólo cambios positivos o negativos y de los que percibieron ambos tipos de cambios. 
bios positivos en el país y no lograron identificar deterioro en el mismo; éstos suman el 27.6 por ciento. En tercer lugar está el otro lado de la moneda: los que sólo percibieron cambios negativos y que llegan al 20 por ciento de la población. Finalmente está el grupo que se constituye en el más numeroso, los que reconocen los cambios positivos pero advierten al mismo tiempo sobre las transformaciones negativas que ha sufrido el país con la segunda gestión arenera; éstos representan casi la tercera parte de los ciudadanos y son los más propensos a evitar las evaluaciones absolutistas con respecto al gobiemo. En todo caso, estos resultados muestran que la máyor parte de la gente está dividida con respecto al legado de Calderón mientras se mantuvo en la presidencia.

\section{Cuadro 5}

Opinión acerca de cómo está el país desde que entró el actual gobierno de ARENA, según variables

(En porcentajes)

\begin{tabular}{llllll}
\hline & & & Opinión & & $\begin{array}{l}\text { No } \\
\text { Sabe }\end{array}$ \\
\cline { 2 - 5 } Variables & Peor & Igual & Mejor & 1.6 \\
\hline Todos & 36.6 & 33.7 & 28.1 & \\
Estrato & & & & 0.0 \\
Alto & 26.3 & 33.7 & 40.0 & 1.1 \\
Medio-alto & 30.9 & 38.3 & 29.7 & 1.7 \\
Medio-bajo & 33.7 & 39.0 & 25.6 & 0.8 \\
Obrero & 41.3 & 30.4 & 27.5 & 0.6 \\
Marginal & 44.1 & 29.8 & 25.5 & 2.9 \\
Rural & 36.8 & 33.2 & 27.2 & \\
Estudios & & & & 3.8 \\
Ninguno & 40.9 & 26.4 & 28.9 & 2.0 \\
Primaria & 42.9 & 33.2 & 21.9 & 1.6 \\
Plan básico & 38.1 & 29.6 & 31.7 & 0.4 \\
Bachillerato & 30.4 & 36.8 & 31.2 & \\
Superior & 29.7 & 38.7 & 31.2 & 1.2 \\
Opción de voto & & & & 1.1 \\
Ninguno & 47.8 & 35.9 & 15.1 & 1.3 \\
ARENA & 21.8 & 33.4 & 43.7 & 2.2 \\
FMLN & 50.0 & 31.8 & 16.9 & 0.0 \\
CDU & 47.8 & 43.5 & 6.5 & 0.0 \\
PDC & 62.1 & 20.7 & 17.2 & 2.8 \\
Otros partidos & 63.6 & 36.4 & 0.0 & 27.0 \\
No sabe, voto secreto & 37.1 & 33.1 & & \\
\hline & & & & \\
\hline
\end{tabular}

En su opinión, ¿el país está mejor, igual o peor que hace cinco años cuando entró el actual gobierno?

Pero cuando se trata de juzgar de forma directa la situación del país con el gobierno arenero, las respuestas tienden a decantarse un poco más por lo negativo, aunque sin una mayoría absoluta. El 36.6 por ciento piensa que el país está peor que hace cinco años cuando ARENA tomó la presidencia por segunda vez; el 33.7 por ciento sostuvo que el país está igual, que no ha mejorado ni ha empeorado; mientras que el 28.1 por ciento afirmó que el país ha mejorado. Como puede verse, la distribución de las respuestas es bastante pareja y muestra opiniones divididas con respecto al impacto de la gestión gubernamental sobre el estado del país. Sin embargo, estas opiniones varían en función de variables como el estrato socieconómico del entrevistado, el nivel educativo y, so- 
bre todo, la alineación política del mismo. La percepción de que el país está peor luego de cinco años de presidencia calderonista es más común en la medida en que los consultados pertenecen a sectores desaventajados socialmente (obreros, marginados y campesinos) y en la medida en que tienen menos formación educativa; además suelen ser más críticos con respecto a la situación del país quienes no son partidarios del partido en el gobierno. Exceptuando esta última condición, los resultados -que se muestran en el Cuadro 5- reiteran el peso de la posición socioeconómica a la hora de valorar al gobiemo y a los aspectos relacionados con él: el apoyo y la visión más favorable hacia el gobierno y hacia sus obras surgen invariablemente de aquéllos sectores mejor ubicados socialmente, y el rechazo o la indiferencia son más frecuentes en el otro extremo del espectro social.

Ahora bien, un dato interesante es el que muestra que las personas que señalan aspectos económicos como problemas fundamentales del país, suelen tener una visión más negativa acerca de la situación que quienes señalan otros tipos de dificultades nacionales. Ello sugeriría que las valoraciones de tipo económico tienen mucho peso a la hora de considerar la situación del país, incluso estarían por encima de la preocupación por la delincuencia. De hecho, cuando se preguntó a los salvadoreños por la situación económica del país con el gobierno de Armando Calderón Sol, las respuestas de carácter pesimista superaron con amplio margen a las optimistas; es decir, cerca de la mitad de los ciudadanos piensa que la situación económica del país ha empeorado con el segundo gobiemo arenero, frente a un 19.4 por ciento que señala lo opuesto y un 39 por ciento que cree que la situación económica del país se mantiene igual. En este caso, las opiniones desfavorables son especialmente frecuentes entre los residentes del AMSS, entre los ciudadanos que pertenecen a los estratos medio-bajo y obrero, entre las personas entre 41 y 55 años de edad y entre los que no han tenido formación escolar.

Sin embargo, las opiniones sobre la situación económica del país no siempre se presentan de la misma manera que las afirmaciones sobre las condiciones económicas familiares particulares. Aunque el balance tiende más hacia lo negativo que hacia lo positivo, el dictamen predominante en este caso es que su situación económica familiar se mantiene sin cambios de consideración. En todo caso, es interesante advertir que los salvadoreños piensan que las condiciones económicas del país han empeorado aunque eso no signifique directamente que sus condiciones económicas particulares se hayan deteriorado de la misma forma; la idea detrás de esto es alg̈o así como: "yo estoy igual (¿de mal?), pero el país sí está peor”. Esto se debe probablemente a que los consultados responden más en función de su percepción pública sobre las expectativas del país que sobre sus propias expectativas de progreso económico.

En todo caso y en términos generales, los datos indican que la mayor parte de los salvadoreños consideran que las condiciones económicas del país no han mejorado, antes bien habrían empeorado con la gestión del segundo gobierno arenero. De ahí que cuando a los ciudadanos se les preguntó si el país va por un buen camino, el 81.8 por ciento haya respondido que necesita un cambio. La mayoría de quienes respondieron de esa forma son aquéllos que piensan que la situación general del país está peor o está igual que hace cinco años. En el fondo, los salvadoreños estaban deseando un cambio en la forma de gobernar.

\section{Figura 3}

Opinión sobre la situación económica del país y familiar con el gobierno de Calderón

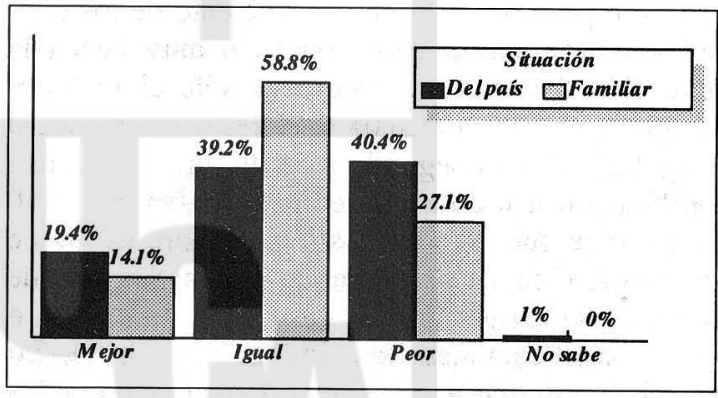

¿Cree usted que la situación económica del país mejoró o empeoró con el actual gobierno?/¿Su situación económica familiar mejoró o empeoró con el actual gobierno?

\section{La evaluación general del gobierno}

Todos los datos anteriores muestran una particularidad: a pesar de que varias de las opiniones tienden más hacia lo negativo que hacia lo positivo, no existe una opinión dominante que se imponga muy por encima de las otras. Los juicios de los salvadoreños aparecen más bien divididos y, 
en muchos aspectos, contradictorios. Por ejemplo, el saldo positivo que dejan las opiniones acerca de los cambios positivos en el país con el gobierno de Calderón encuentra su contrapeso en las opiniones que dicen que la nación no está mejor que hace cinco años. Buena parte de los salvadoreños piensa que el gobierno sólo se preocupó por los más ricos, pero la mayoría de ciudadanos admite que sus condiciones económicas particulares no empeoraron durante la segunda presidencia arenera.

Ésta es una característica que se traslucirá también en los resultados sobre la evaluación global del gobierno. El sondeo de evaluación gubernamental pidió a los ciudadanos que valoraran el desempeño de la gestión mediante dos formas. Por una lado se les pidió que opinaran sobre la actuación del presidente y que la adjetivaran como buena o mala; y por el otro se les solicitó que calificaran el desempeño del gobierno en general utilizando una escala de 0 a 10 , en donde 0 sería la peor calificación y 10 la mejor.

Con el primer método, el balance de la evaluación fue positivo: casi el 50 por ciento de los consultados calificaron como buena o muy buena la actuación del presidente saliente, sólo el 15.6 por ciento la calificó de mala y el resto la consideró "regular". Con el segundo método, que aplica una calificación a todo su desempeño gubernamental, el balance fue menos positivo: el promedio de evaluación de todas las notas asignadas fue de 5.89; sin embargo, en este caso el 43.7 por ciento de las calificaciones caen por encima del 6. Lo anterior sugiere dos cosas: en primer lugar, que los salvadoreños califican mejor al presidente como persona que al gobiemo mismo; y, en segundo lugar, que las opiniones continúan divididas con respecto al desempeño gubernamental.

Ahora bien, un análisis más detallado de los datos anteriores muestra que la gente suele darle un peso considerable a la percepción de cambios positivos en el país a la hora de realizar la evaluación global del desempeño del gobiemo. Es decir, aquellas personas que percibieron cambios positivos en el país, a pesar de que hayan percibido cambios negativos también, están más propensas a calificar como bueno o a asignar una nota por encima de 6 al gobierno en lugar de hacer lo contrario. He aquí el peso de la construcción de obras de infraestructura, las cuales tienen un impacto positivo sobre la opinión pública. Pero de acuerdo con los datos, la variable que tiene más peso a la hora de determinar la valoración global del gobierno es la percepción sobre la capacidad del mismo para gobernar. Los resultados muestran que casi la mitad de los salvadoreños (49.1 por ciento) opinaron que el gobierno de Calderón Sol tuvo algo o mucha capacidad para conducir al país, mientras que la otra mitad sostuvo que el gobierno tuvo poca o ninguna capacidad para hacerlo; estas opiniones se correlacionaron fuertemente con la calificación general de la administración, de tal manera que en la medida que aumentaba la opinión sobre la capacidad del gobierno, en esa medida aumentó también la calificación global del mismo.

Esto no sucedió de la misma manera con las opiniones sobre la transparencia y honestidad del gobierno. A los salvadoreños se les preguntó sobre qué tan corrupto creían que había sido el gobiemo: el 28.2 por ciento respondió que mucho; el 25.7 por ciento dijo que "algo corrupto", y el 34.1 por ciento manifestó que poco o nada corrupto. El análisis de estas cifras indica que aunque hay cierta relación entre estas opiniones y la evaluación general del gobierno, el peso de las mismas es muy poco a la hora de determinar la calificación del gobierno ${ }^{5}$. En otras palabras, a la hora de evaluar al gobierno, los salvadoreños no otorgan mucha importancia a su imagen de transparencia; para ellos es mucho más importante la imagen de capacidad para conducir al país.

5. Tanto en este caso como en el anterior, el análisis utilizado fue una regresión, la cual permitía establecer el nivel de responsabilidad de cada una de las variables en las valoraciones generales sobre el gobierno. 


\begin{tabular}{lcc}
\multicolumn{3}{c}{$\begin{array}{c}\text { Cuadro } 6 \\
\text { Evaluación del desempeño del gobierno de } \\
\text { Armando Calderón Sol, según variables }\end{array}$} \\
\hline Variables & $\mathrm{N}$ & Promedio \\
\hline Todos & 1207 & 5.89 \\
& & \\
Estrato & & \\
Alto & 91 & 6.48 \\
Medio-alto & 172 & 6.03 \\
Medio-bajo & 167 & 5.87 \\
Obrero & 237 & 5.62 \\
Marginal & 154 & 5.77 \\
Rural & 386 & 5.90 \\
& & \\
Estudios & & \\
Ninguno & 138 & 5.65 \\
Primaria & 368 & 5.73 \\
Plan básico & 186 & 5.98 \\
Bachillerato & 239 & 6.17 \\
Superior & 276 & 5.91 \\
& & \\
Opción de voto & & \\
Ninguno & 240 & 4.68 \\
ARENA & 431 & 7.08 \\
FMLN & 153 & 4.76 \\
CDU & 44 & 5.18 \\
Otros partidos & 39 & 4.89 \\
No sabe, voto secreto & 300 & 5.94 \\
\hline En una escala de a & & \\
\hline
\end{tabular}

En una escala de 0 a 10, ¿qué nota le daría al gobierno de Calderón Sol por su desempeño en estos cinco años? mación educativa, aunque en este caso el efecto contrario no se da entre los más educados, las cifras reiteran la asociación entre desventajas sociales y opinión negativa con respecto al gobierno. Finalmente, el grupo que mejor evaluó al gobiemo por encima de cualquier otro grupo es el que está compuesto por simpatizantes y correligionarios de ARENA, en tanto que los simpatizantes de otros partidos, y sobre todo los que no tienen partido de preferencia, fueron los que peor evaluaron al gobierno.

\section{Figura 4}

La evaluación del gobierno de Calderón Sol a lo largo de su gestión

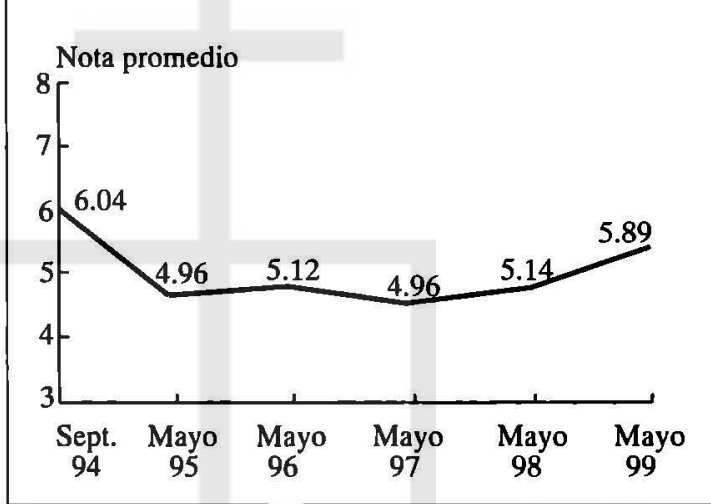

Fuente: IUDOP, boletín de prensa, Año XII, No. 4.

La evaluación global de la gestión calderonista revela, con todo, que la opinión sobre la presidencia mejoró de manera sustancial si se la compara

Ahora bien, ¿quiénes evalúan mejor al gobierno saliente por su desempeño? Después de haber pasado lista a los resultados anteriores, las cifras expuestas en el Cuadro 6 no son sorprendentes, pues siguen las mismas tendencias. La gestión de la segunda presidencia de ARENA encuentra más apoyo en la medida en que las personas pertenecen a un estrato socioeconómico más elevado; de tal manera que las personas que pertenecen a los sectores alto y medio-alto aprueban más el desempeño del gobierno que acaba de concluir, mientras que las personas pertenecientes a los sectores obreros y marginales lo desaprueban más. Por otro lado, el gobierno encuentra menos respaldo entre las personas que tienen ninguna o muy poca for-

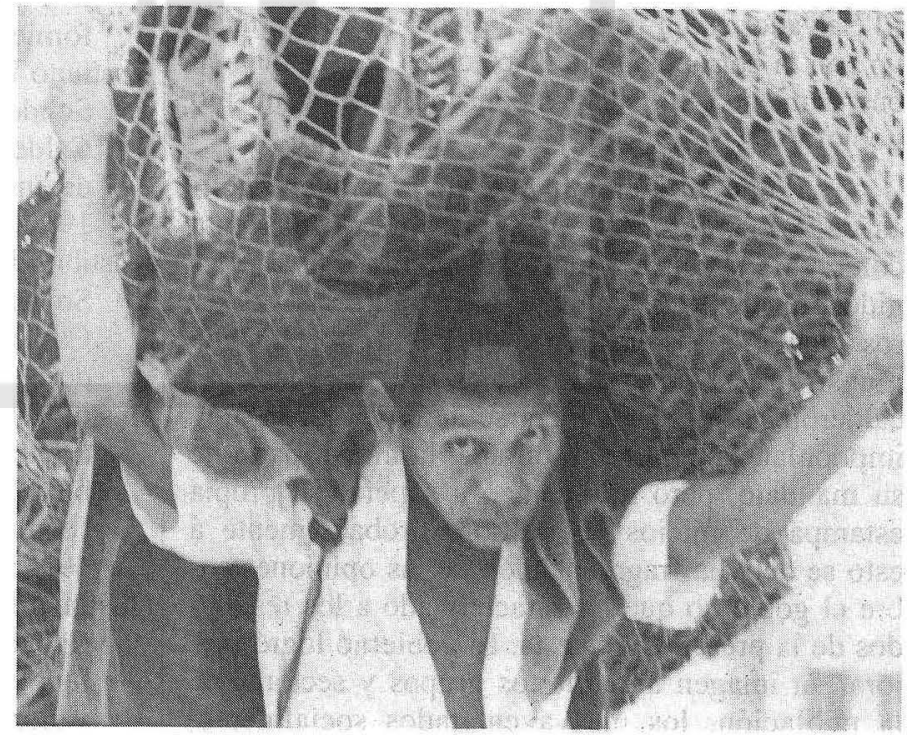


con las evaluaciones anuales anteriores. El gobierno de Armando Calderón Sol no sólo logró mejorar su calificación general de valoración, sino que además mejoró en otras áreas de la opinión pública. Por ejemplo, el porcentaje de personas que encontraron cambios positivos en el país aumentó, mientras que el porcentaje de quienes señalaron cambios negativos disminuyó. La identificación de los logros ascendió, aunque sin agregar algo novedoso (la infraestructura y el sistema educativo fueron señalados en casi toda la gestión); en tanto que la identificación de los fracasos fue mucho menor y más dispersa. El porcentaje de personas que piensan que el país empeoró con la segunda tanda de ARENA sigue siendo alto tanto a nivel general como a nivel económico, pero es menor que los porcentajes obtenidos durante la mayor parte de la gestión. Mucha gente piensa que el presidente no pudo cumplir con todas sus promesas, pero esta proporción de personas es sustancialmente menor a cualquier cifra obtenida anteriormente. Probablemente el mayor logro que se trasluce de las encuestas $-y$ que no aparece tan reconocido conscientemente por la poblacióntiene que ver con la disminución de las preocupaciones ciudadanas por los problemas económicos; aunque éstos no han dejado de existir, la proporción de gente que los advierte es mucho menor que hace cinco años, sobre todo en el área del costo de la vida. Donde no parece haber mejorado sustantivamente el gobiemo, de acuerdo con las distintas opiniones, es en lo referente al combate contra la delincuencia y la corrupción; por el contrario, los salvadoreños creen que al final del mandato calderonista, El Salvador es un país más violento y más permisivo con la compción que hace cinco años y que, en buena medida, ha sido responsabilidad del mismo gobiemo.

Con todo, el mejoramiento de la imagen pública de Armando Calderón Sol no fue lo suficiente como para alcanzar los niveles recibidos al ser elegido presidente o inclusive al cumplir sus primeros cien días de gestión (ver Figura 4). Lo que muestran estas tendencias es que el gobierno calderonista logró recuperarse de una imagen muy impopular, la cual reinó durante la mayor parte de su mandato, pero no alcanzó a superar su propia estampa de inicios de gestión. Probablemente a esto se debe la fragmentación de las opiniones sobre el gobierno que ha caracterizado a los resultados de la presente encuesta. El gobierno logró mejorar su imagen ante ciertos grupos y sectores de la población, los más aventajados socialmente, pero no logró hacerlo con el resto de la sociedad que se quedó reclamando sus políticas preferenciales hacia los más poderosos.

Al final, la opinión de los salvadoreños sobre el segundo gobierno arenero parece moverse entre la calificación de "regular" y buena como producto de un sensible esfuerzo por promocionar su imagen pública, a través de la presentación pública de grandes obras y de discursos de eficiencia. Sin embargo, el mayor defecto del gobierno, además de los ya señalados anteriormente, parece haber sido su incapacidad para establecer comunicación y atender las demandas de la población; los salvadoreños, sobre todos los más pobres y desaventajados, se sintieron igual de marginados que siempre con respecto al país. Esto probablemente le cueste al presidente saliente ser considerado no más allá del primer presidente de la era de posguerra.

\section{Bibliografia}

Coleman, K.M., Cruz; J.M., y Moore, P.J. "Retos para consolidar la democracia en El Salvador". Estudios Centroamericanos (ECA), 571-572, 1996, pp. 415-440.

Instituto Universitario de Opinión Pública (IUDOP). Encuesta de evaluación del gobiemo, alcaldías y Asamblea Legislativa 1999. Serie de informes 76. San Salvador: IUDOP-UCA, 1999.

Instituto Universitario de Opinión Pública (IUDOP). Evaluación del cuarto año de gobierno de Calderón y el primer año de la Asamblea Legislativa y los gobiernos municipales. Serie de informes 69. San Salvador: IUDOP-UCA, 1998.

Instituto Universitario de Opinión Pública (IUDOP). Sondeo de evaluación sobre el tercer año de Calderón Sol y evaluación post-electoral. Serie de informes 63. San Salvador: IUDOP-UCA, 1997.

Instituto Universitario de Opinión Pública (IUDOP). Sondeo de evaluación sobre el segundo año de Calderón Sol. Serie de informes 55. San Salvador: IUDOP-UCA, 1996.

Instituto Universitario de Opinión Pública (IUDOP). Encuesta de evaluación del primer año de gobierno de Armando Calderón Sol. Serie de informes 48. San Salvador: IUDOP-UCA, $1995 a$.

Instituto Universitario de Opinión Pública (IUDOP). Encuesta sobre cultura política, plan y coyuntura económica. Serie de informes 46. San Salvador: IUDOP-UCA, $1995 b$. 
Instituto Universitario de Opinión Pública (IUDOP). Las expectativas de la población hacia el nue- vo gobiemo de ARENA. Boletín de prensa. Año IX, No. 3. San Salvador, 1994.

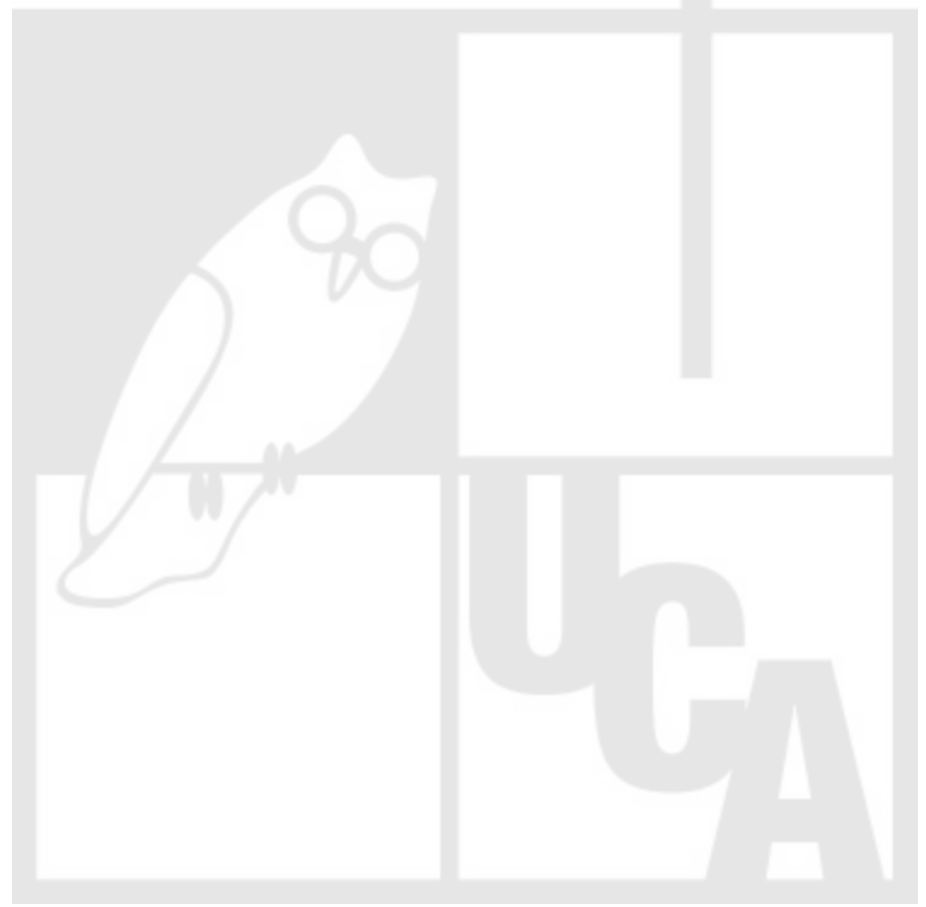

EL GOBIERNO DE CALDERÓN SOL

505

Digitalizado por Biblioteca "P. Florentino Idoate, S.J."

Universidad Centroamericana "José Simeón Cañas" 